Arq. Bras. Med. Vet. Zootec., v.56, n.1, p.86-93, 2004

\title{
Indicadores de desempenho em rebanho da raça Holandesa: curvas de crescimento e altura, características reprodutivas, produtivas e parâmetros genéticos
}

\author{
[Productive indicator in a Holstein dairy herd: growth and height curves, reproductive \\ and productive traits and genetic parameters] \\ J.E. Val ${ }^{1}$, M.A.R. Freitas ${ }^{1 *}$, H.N. Oliveira ${ }^{2}$, V.L. Cardoso $^{3}$, P.F. Machado ${ }^{4}$, J.C.C. Paneto ${ }^{1}$ \\ ${ }^{1}$ Departamento de Genética, FMRP - USP-Ribeirão Preto, SP \\ ${ }^{2}$ FMVZ - UNESP- Botucatu, SP \\ ${ }^{3}$ Instituto de Zootecnia - Ribeirão Preto, SP \\ ${ }^{4}$ ESALQ-USP - Piracicaba, SP
}

\begin{abstract}
RESUMO
Foram analisados dados de peso, altura e desempenho produtivo e reprodutivo de 1.272 novilhas da raça Holandesa, nascidas no período de 1990 a 1998. Estudaram-se o desenvolvimento em peso e altura do nascimento até o primeiro parto, as características peso ao primeiro parto (PP), idade ao primeiro parto (IP), produção de leite na primeira lactação (PL), primeiro intervalo de partos (IEP), e os parâmetros genéticos pertinentes. As equações para as curvas de peso e altura, estimadas pelo modelo proposto por von Bertalanffy, foram: Yi= 802,10 (1 - 0,630EXP (-0,0022t)) e Yi=1,40 (1 - 0,208EXP (-0,0038t)), respectivamente. As novilhas atingiram o primeiro parto com altura média de $140 \mathrm{~cm}$ e peso de $602 \mathrm{~kg}$ aos 27 meses de idade. A produção total de leite na primeira lactação foi de $8.026 \mathrm{~kg}$ e o primeiro IEP 420 dias. Na obtenção dos componentes de (co)variância utilizou-se o método da máxima verossimilhança restrita livre de derivada. As estimativas de herdabilidade foram 0,31,0,35 e 0,34, respectivamente, para PP, IP e PL, e 0,10 para IEP, evidenciando variância genética aditiva média para as três primeiras características. As correlações genéticas apresentaram pequena magnitude para PP e PL, PL e IP, e IEP e PL. A correlação entre IEP e PP foi 0,49; entre IEP e IP o valor foi negativo, -0,69, indicando provável antagonismo entre as duas últimas características.
\end{abstract}

Palavras-chave: sistema de produção de leite, peso, herdabilidade, correlação genética

\begin{abstract}
Data on weight, height, productive and reproductive performance of 1,272 Holstein heifers, born from 1990 to 1998, were used to evaluate the growth and height patterns of these females from birth to first calving, and the following traits: weight at first calving (PP), age at first calving (IP), milk production in the first lactation $(P L)$ and first calving interval (IEP), and to estimate genetic parameters of these traits. The von Bertalanffy fitted models were: Yi=802.10 (1-0.630EXP (-0.0022t)) for weight, and Yi=1.40 (1$0.208 \mathrm{EXP}(-0.0038 \mathrm{t})$ ) for height. The average performance of heifers were: height $140 \mathrm{~cm}$, weight $602 \mathrm{~kg}$ and 27 month of age at first calving. The total milk yield was $8,026 \mathrm{~kg}$ and first IEP was 420 days. The (co)variance components estimates were obtained by a derivative-free algorithm with the DFREML. The estimates of heritabilities were 0.31, 0.35, 0.34, respectively for PP, IP and PL, and 0.10 for IEP. The genetic correlations estimates between PP and PL, PL and IP and IEP and PL were low. The correlation
\end{abstract}

Recebido para publicação em 7 de dezembro de 2002

Recebido para publicação, após modificações, em 5 de dezembro de 2003

*Autor para correspondência:

Rua Lafaiette, 1222, apto. 72

14015-080 - Ribeirão Preto

E-mail: mfreitas@genbov.fmrp.usp.br 
between IEP and PP was 0.49 and between IEP and IP was, -0.69, sugesting an antagonism between the two last traits.

Keywords: milk production system, weight, heritability, genetic correlation

\section{INTRODUÇÃO}

Estudos sobre o desenvolvimento ponderal nos bovinos e em especial sobre as curvas de crescimento das raças leiteiras são relativamente escassos no Brasil. Entre os mais recentes estão os de Oliveira (1994) e Perotto et al. (1997). Para Oliveira (1995), o crescimento pode ser expresso em termos numéricos, sendo, portanto, passível de análises quantitativas. Quando se obtêm pesagens subseqüentes e regulares de um mesmo indivíduo, do nascimento à maturidade, é possível construir uma curva de peso em função da idade para representar o crescimento. Devido à grande quantidade de dados gerados durante $\mathrm{o}$ crescimento podem surgir dificuldades de interpretação, entretanto, o uso de modelos estatísticos apropriados permite condensá-los em poucos parâmetros como, por exemplo, os citados no modelo de von Bertalanffy. Assim, a informação matemática contida em tais dados e sua interpretação biológica é adequada.

Segundo Oliveira (1995), os modelos mais usados para descrever o crescimento são os não lineares, do tipo sigmóide, como os modelos de Richards, Brody e von Bertalanffy. Perotto et al. (1992), trabalhando com rebanho das raças Holandesa e Aryshire, concluíram que o modelo de Richards mostrou melhor ajuste para os dados quando comparados com os modelos de Brody e von Bertalanffy. Para Koenen e Groen (1995), em estudo semelhante na Europa com a raça Holandesa, o modelo de von Bertalanffy ajustou melhor os dados em relação a outros modelos. Obtiveram estimativas de $667,0,586$ e 0,00269 para os parâmetros A, B e k, respectivamente.

A identificação precoce de animais superiores geneticamente é fator decisivo do processo de produção, uma vez que a eficiência dos sistemas é medida pela taxa de progresso obtido por unidade de tempo. Além da produção de leite, características como peso elevado ao primeiro parto, precocidade ao primeiro parto e intervalo de partos curtos são importantes para aumentar a produção total na vida útil do animal. Essas características visam, principalmente, medir a capacidade reprodutiva dos animais que, se melhoradas, propiciam substituição mais rápida do material genético na população pela conseqüente redução do intervalo de gerações (Grossi, 1999; Zambianchi, et al. 1999).

Com relação à importância das características envolvendo pesos do nascimento ao parto nos bovinos leiteiros, Groen e Vos (1994) obtiveram valores de herdabilidade $\left(\mathrm{h}^{2}\right)$ de 0,46 a 0,64 para peso corporal, 0,43 para o crescimento do nascimento até 50 semanas de idade e 0,14 para pesos da inseminação até o parto. A correlação genética $\left(\mathrm{r}_{\mathrm{g}}\right)$ entre pesos nas várias idades variou de 0,42 a 1,0. Em outro estudo (Koenen, Groen, 1995), os valores de $h^{2}$ para peso ao parto variaram de 0,48 a 0,60 . Essa característica apresentou alta $r_{g}, 0,74$ a 0,90 , com peso corporal à maturidade.

No processo de seleção dos bovinos leiteiros, a idade ao primeiro parto é uma característica que deve ser considerada. Ela pode ser reduzida pela seleção por apresentar valores de $h^{2}$ de médio a alto. O manejo precisa ser corretamente definido, pois diversos fatores ligados ao ambiente afetam o crescimento animal, influenciando a expressão da característica (Pereira, 2001).

No Brasil, Cardoso (1985) obteve $\mathrm{h}^{2}$ igual a 0,57 para idade ao primeiro parto em animais da raça Holandesa. Freitas et al. (2000), em estudo com a mesma raça em Minas Gerais, obtiveram média para idade ao primeiro parto de $27,5 \pm 5$ meses e valor de herdabilidade de $0,27 \pm 0,05$.

Para Deshmukh et al. (1993), idade ao primeiro parto, produção de leite na primeira lactação e produção de leite por dia de intervalo de partos seriam critérios úteis para comparar o mérito econômico nos rebanhos leiteiros.

Nos Estados Unidos, Albuquerque et al. (1996) e Gadini et al. (1997) mostraram valores de $\mathrm{h}^{2}$ de 0,34 e 0,19 para produção de leite na primeira lactação, demonstrando a variação existente para a característica. No Brasil, Machado et al. (1999), ao estudarem dados de produção de leite 
na primeira lactação, de vários rebanhos da raça Holandesa, criados predominantemente na Região Sudeste do País, obtiveram estimativas de $\mathrm{h}^{2}$ de 0,22 a 0,35 , dependendo do modelo de análise efetuada.

Os principais objetivos deste estudo foram analisar o desenvolvimento em peso e altura de fêmeas da raça Holandesa até o primeiro parto, descrever os coeficientes técnicos obtidos e estimar os parâmetros genéticos $\left(h^{2}\right.$ e $\left.r_{g}\right)$ para peso ao primeiro parto, idade ao primeiro parto, produção de leite na primeira lactação e primeiro intervalo de partos.

\section{MATERIAL E MÉTODOS}

Os dados utilizados pertencem a fêmeas da raça Holandesa, oriundos de uma propriedade no Estado de São Paulo, que participa do Programa de Análise de Rebanhos Leiteiros e da Qualidade do Leite, conduzido pelo Departamento de Produção Animal/ ESALQ/USP-SP. O estudo incluir todas as fêmeas nascidas no período de 1990 a 1998. O sistema de produção é intensivo e a alimentação é feita pela suplementação com silagem de milho e ração balanceada, de acordo com o nível de produção de leite. A partir de 1996 foi adotado o sistema de dieta total ou completa. As bezerras, filhas de touros provados para produção de leite, são separadas das mães após a ingestão do colostro.

As fêmeas entram em reprodução ao redor do $14^{\circ}$ mês de idade ou quando atingem cerca de $380 \mathrm{~kg}$ de peso e $130 \mathrm{~cm}$ de altura. A fazenda adota o sistema de ordenha mecânica, realizada três vezes ao dia.

Para análise do crescimento em peso e altura individual médio das fêmeas, foi adotado o modelo de curva proposto por von Bertalanffy (1957):

$\mathrm{Yt}=\mathrm{A}\left(1-\mathrm{Be}^{-\mathrm{kt}}\right)+\varepsilon$, em que:

$\mathrm{Yt}=$ peso do animal na idade $\mathrm{t} ; \mathrm{A}=$ valor assintótico de Yt (peso médio do animal adulto); $\mathrm{k}=$ taxa de variação da função exponencial (fornece uma perspectiva da precocidade); $\mathrm{e}=$ base do logaritmo natural; $\mathrm{B}=$ constante de integração relacionada com os pesos iniciais; $\varepsilon=$ erro aleatório associado a cada pesagem.
Com relação à organização, formação e consistência dos arquivos, análises descritivas e análises de variância para efeitos não genéticos foram usados os procedimentos disponíveis no Statistycal Analysis System (User's..., 1995). As variáveis usadas no arquivo final foram: identificação do animal $(\mathrm{N})$; grupo contemporâneo formado por ano e estação de parto (estação chuvosa de outubro a março e estação seca de abril a setembro) (ANOEST); intervalo entre o parto e a pesagem mais próxima (IPAPE), variável incluída por não se dispor das medidas padronizadas ao parto, sendo então necessário corrigir as diferenças entre o peso observado e o peso ao parto; duração da lactação (DEL); idade ao primeiro parto (IP); peso ao parto (PP); produção total de leite na primeira lactação (PL); e primeiro intervalo de parto (IEP). Este arquivo correspondeu a informações de 1.272 animais.

Para estimar os parâmetros genéticos após foram usadas informações de 839 novilhas, filhas de 100 touros.

Para obtenção das estimativas de $h^{2}$ e $r_{g}$ das características PP, IP, PL e IEP foi formado um arquivo de parentesco, além daquele que continha os dados referentes às características produtivas e reprodutivas. Para essa análise, foi utilizado o método da máxima verossimilhança restrita livre de derivada (DFREML), sob modelo animal por meio do software multiple trait derivative-free restricted maximum likeliwood, descrito por Boldman et al. (1995), sob o seguinte modelo na forma matricial:

$\mathrm{Y}=\mathrm{X} \beta+\mathrm{Zv}+\mathrm{e}$, em que:

$Y=$ vetor de informação; $\beta=$ vetor de efeitos fixos; $X=$ matriz de incidência que relaciona $\beta$ com $Y ; v=$ vetor dos efeitos aleatórios (genético aditivo); $Z=$ matriz de incidência que associa elementos de $\mathrm{v}$ com $\mathrm{Y}$; $\mathrm{e}=$ vetor dos efeitos aleatórios residuais, de acordo com as pressuposições: $\mathrm{V}(\mathrm{v})=\mathrm{G}=\mathrm{A} \sigma^{2} \mathrm{a} ; \mathrm{V}(\mathrm{e})=\mathrm{R}=$ $\mathrm{I} \sigma^{2} \mathrm{e} ; \operatorname{Cov}\left(\mathrm{v}, \mathrm{e}^{\prime}\right)=0$

As correlações genéticas foram obtidas por meio de análises bi-variadas, incluindo os seguintes pares de características: PP e PL; IEP e IP; PL e IP; IEP e PP e IEP e PL. 


\section{RESULTADOS E DISCUSSÃO}

Os parâmetros médios das curvas de peso e altura estimados, de acordo com o modelo proposto por Von Bertalanffy, constituíram as seguintes equações: $\mathrm{Yi}=802,10(1-0,630 \mathrm{EXP}(-$ $0,0022 \mathrm{t}))$ e $\mathrm{Yi}=1,40(1-0,208 \mathrm{EXP}(-0,0038 \mathrm{t}))$, respectivamente. $\mathrm{O}$ valor de A para peso deve ser analisado com reserva, visto que os pesos são mais numerosos até os primeiros 800 dias de idade, pois a partir do primeiro parto a fazenda adota o critério de fazer as pesagens das fêmeas apenas por ocasião das parições subseqüentes. $\mathrm{O}$ peso adulto de $802 \mathrm{~kg}$ foi superior ao resultado obtido por Koenen e Groen (1995), de 667kg, em estudo com a mesma raça. O manejo nutricional adotado pela propriedade, aliado ao potencial genético dos animais para ganho de peso nas diversas fases do crescimento, pode ter contribuído para o valor obtido neste estudo.

Com relação ao parâmetro $\mathrm{B}$, o valor de $0,630 \mathrm{~kg}$ confirma o bom desempenho do rebanho quanto ao ganho de peso, mostrando que apesar da deficiência de dados dos pesos após os 800 dias de idade, foi possível delinear o comportamento médio do crescimento dos animais.

Na Tab. 1 são apresentadas as médias estimadas para os pesos nas diferentes idades, ajustadas segundo o modelo de von Bertalanffy.

Tabela 1. Média dos pesos $(\mathrm{kg})$ estimadas no nascimento (PN), aos 60 dias (P60), aos 240 dias (P240), aos 360 dias (P360), aos 600 dias (P600), aos 660 (P660) e 720 dias (P720) de idade em fêmeas da raça Holandesa segundo modelo de equação proposto por von Bertalanffy

\begin{tabular}{ccccccc}
\hline PN & P60 & P240 & P360 & P600 & P660 & P720 \\
\hline 40,4 & 72,4 & 202,1 & 297,5 & 467,9 & 503,6 & 536,2 \\
\hline
\end{tabular}

Na Fig.1 observam-se as curvas de peso e altura das novilhas, obtidas a partir das equações citadas. Verifica-se que os animais cresceram bem, atingindo peso e altura adequados nas diversas fases do desenvolvimento.

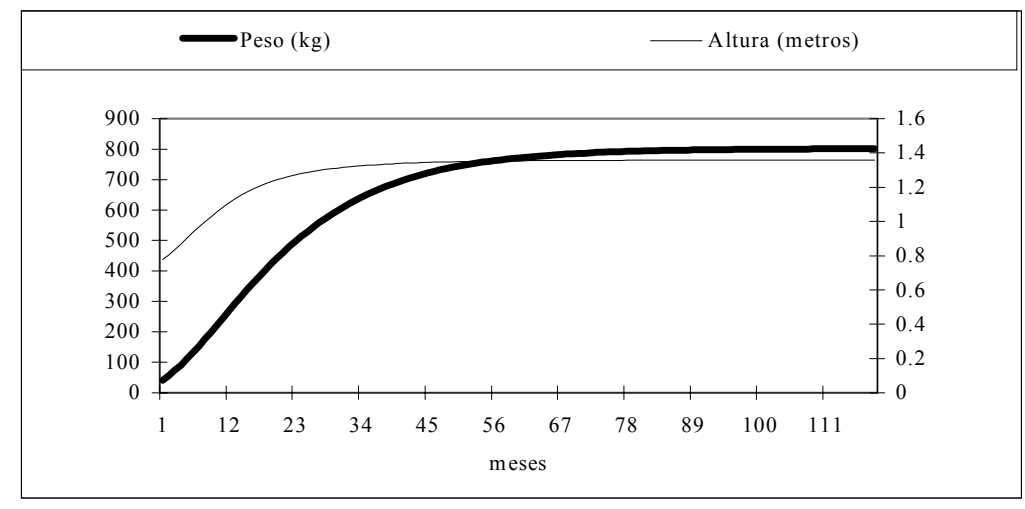

Figura 1. Curvas de peso e altura em rebanho da raça Holandesa.

Com referência aos coeficientes técnicos de desempenho produtivo e reprodutivo (Tab. 2), verifica-se que, em média, as novilhas conceberam aos 532 dias de idade (17,5 meses), cresceram $10 \mathrm{~cm}$ e ganharam $0,664 \mathrm{~kg}$ por dia da concepção ao parto, atingiram $140 \mathrm{~cm}$ de altura e $602 \mathrm{~kg}$ de peso ao primeiro parto, que ocorreu, em média, aos 800 dias de idade (aproximadamente 26 meses). Esses ganhos de peso são próximos dos citados por van Amburgh et al. (1998) em estudo com a mesma raça. O manejo do rebanho é conduzido de forma que as novilhas atinjam peso e altura para início da vida reprodutiva o mais precoce possível. $\mathrm{O}$ intervalo médio entre o primeiro e segundo partos, 420 dias, é bom considerando-se o nível de produção do rebanho. Este valor é semelhante ao citado por Silva (1998), em estudos com a mesma raça. 
Deve-se considerar que existe tendência por parte dos produtores em retardar a inseminação após o primeiro parto, principalmente de novilhas com alto potencial de produção de leite e mais persistentes do rebanho. Assim, o valor do IEP pode ser considerado bom tendo em vista o nível de produção de leite do rebanho na primeira lactação, $8.026 \mathrm{~kg}$, com duração média da lactação de 346 dias, resultado expressivo quando comparado com a média nacional, de $7.000 \mathrm{~kg}$ para a raça Holandesa.
A Fig. 2 apresenta a evolução das médias de produção de leite na primeira lactação de acordo com o ano. Os valores mais baixo e mais alto foram 6.951 e $9.165 \mathrm{~kg}$, obtidos em 1992 e 1998 , respectivamente. $\mathrm{O}$ aumento foi mais evidente a partir de 1995, quando o rebanho foi incluído no Programa de Gerenciamento de Rebanhos Leiteiros e da Qualidade do Leite da ESALQ/USP.

Tabela 2. Número de observações $(\mathrm{N})$, média observada, desvio-padrão (DP) e coeficiente de variação (CV) para as características em estudo

\begin{tabular}{lcccc}
\hline Variável & $\mathrm{N}$ & Média & $\mathrm{DP}$ & $\mathrm{CV} \%$ \\
\hline Altura à concepção (m) & 1136 & 1,30 & 0,14 & 11,13 \\
Peso à concepção (Kg) & 1136 & 421,06 & 43,65 & 10,36 \\
Idade à concepção (dias) & 1136 & 532,79 & 75,60 & 14,18 \\
Altura ao parto (m) & 940 & 1,40 & 0,04 & 2,81 \\
Peso ao parto (Kg) & 839 & 602,80 & 52,20 & 8,66 \\
Idade ao parto (dias) & 946 & 800,64 & 86,64 & 10,82 \\
Produção de leite (kg) & 836 & $8.026,01$ & $2.511,59$ & 31,00 \\
Duração da lactação (dias) & 836 & 346,43 & 84,55 & 31,29 \\
Intervalo de parto (dias) & 657 & 420,72 & 95,23 & 22,63 \\
\hline
\end{tabular}

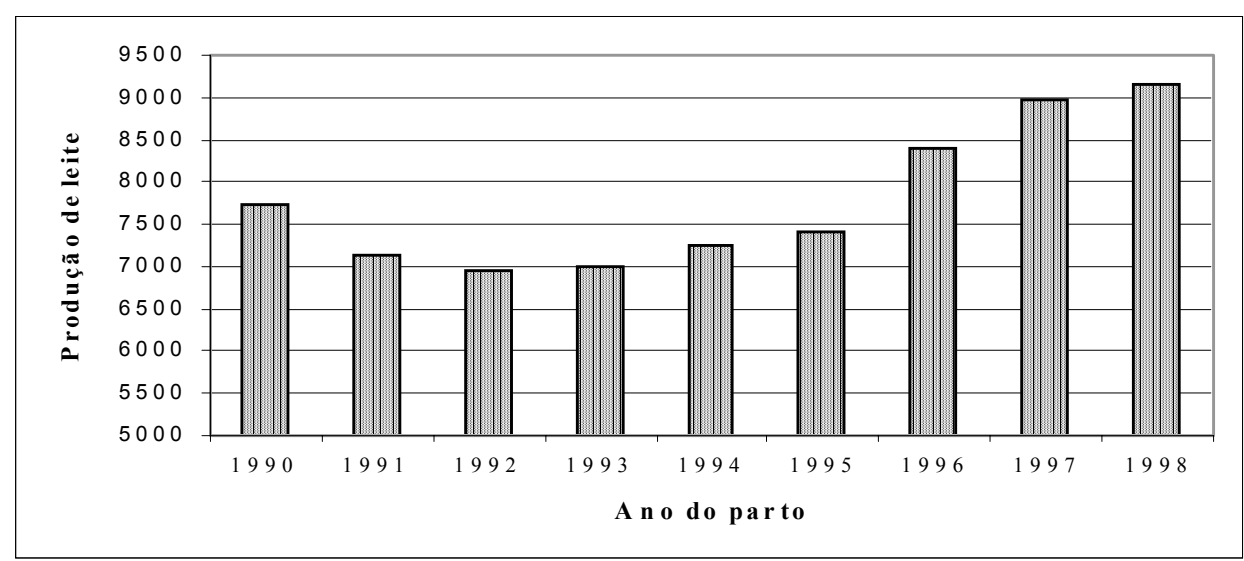

Figura 2. Evolução da produção de leite do rebanho no período de 1990 a 1998.

$\mathrm{O}$ efeito ANOEST foi significativo sobre as características analisadas $(\mathrm{P}<0,01)$, exceto para PL. Ao serem analisados separadamente os efeitos de ano e de estação do parto sobre a produção de leite (PL), somente ano do parto foi significativo, provavelmente devido às condições de alimentação e manejo oferecidas às novilhas em lactação. Quanto ao PP, IP e IEP, os resultados sugerem que essas características podem ser mais sensíveis às diferenças de ambiente.

Os efeitos linear e quadrático do PP foram significativos sobre a IP, o mesmo acontecendo com o efeito da PL sobre o IEP. 
As estimativas de $\mathrm{h}^{2}$ foram de $0,31,0,35,0,34 \mathrm{e}$ 0,10 , respectivamente, para $\mathrm{PP}$, IP, PL e IEP (Tab. 3). O coeficiente obtido para PP foi inferior aos 0,64 e 0,48 citados por Groen e Vos (1994) e Koenen e Groen (1995), respectivamente. Esses autores também analisaram dados de apenas um rebanho, porém, tinham medidas padronizadas de peso ao parto. Nesta pesquisa utilizaram-se pesagens mais próximas ao parto e procurou-se corrigi-las incluindo a covariável IPAPE no modelo estatístico.

Tabela 3. Coeficientes de herdabilidade e correlações genéticas, segundo análises bivariadas para PP (PL), IEP (IP), PL (IP), IEP (PP) e IEP (PL)

\begin{tabular}{llll}
\hline Características & $\mathrm{h}_{\mathrm{a} 1}^{2}$ & $\mathrm{~h}_{\mathrm{a} 2}^{2}$ & $\mathrm{r}_{\mathrm{a} 1 \mathrm{a} 2}$ \\
\hline PP (PL) & 0,31 & 0,34 & $-0,03$ \\
IEP (IP) & 0,10 & 0,35 & $-0,69$ \\
PL (IP) & 0,34 & 0,35 & $-0,20$ \\
IEP (PP) & 0,10 & 0,28 & 0,49 \\
IEP (PL) & 0,08 & 0,33 & $-0,26$ \\
\hline
\end{tabular}

$\mathrm{h}^{2}{ }_{\mathrm{a} 1}=$ herdabilidade da característica $1 ; \mathrm{h}_{\mathrm{a} 2}^{2}=$ herdabilidade da característica $2 ; \mathrm{r}_{\mathrm{ala2}}=$ correlação genética. $\mathrm{PP}=$ idade ao primeiro parto; $\mathrm{PL}=$ produção de leito ao primeiro parto; $I E P=$ intervalo entre primeiro e o segundo parto; $I P=$ idade ao primeiro parto

$\mathrm{O}$ valor de $\mathrm{h}^{2}$ para IP é semelhante aos citados por Grossi (1999) e Freitas et al. (2000). A IP pode auxiliar na seleção direta para outras características de crescimento e de reprodução, visto ser ela a primeira etapa a cumprir, quando se deseja melhorar a eficiência reprodutiva dos rebanhos. Atenção à IP é importante graças a sua relação com a precocidade e rentabilidade do processo produtivo.

Com relação à estimativa de $\mathrm{h}^{2}$ para a $\mathrm{PL}$, os valores foram semelhantes àqueles citados por Albuquerque et al. (1996) e Freitas et al. (2000) e superiores aos obtidos por Dong e Van Vleck (1989) e Gadini et al. (1997), confirmando ser a característica passível de seleção em programas de melhoramento genético dos bovinos leiteiros.

As estimativas de $h^{2}$ obtidas pare IEP situaramse entre as mencionadas por vários autores, 0,08 a 0,10 (Silva et al., 1998; Grossi, 1999; Zambianchi et al., 1999), valores relativamente baixos, confirmando ser a característica predominantemente influenciada pelos efeitos ambientais.
O baixo valor encontrado para a $r_{g}$ entre PP e PL sugere que a seleção para a primeira não influencia a seleção para a segunda característica, aspecto já abordado por Oliveira et al. (1994).

A estimativa de $r_{g}$ mais expressiva foi entre IEP e IP $(-0,69)$, sugerindo provável antagonismo na seleção para as duas características. Assim, seleção para animais precoces ao primeiro parto poder estar contribuindo para o aumento do primeiro intervalo de partos.

A $r_{g}$ entre IP e PL, cuja magnitude foi relativamente baixa $(-0,20)$, sugere que a seleção para redução da idade ao primeiro parto pouco afeta a seleção para produção de leite na primeira lactação. No entanto, é importante considerar que animais que iniciaram a vida reprodutiva demasiadamente cedo, se não estiverem em bom estado nutricional, podem também apresentar reduzida a produção de leite na primeira lactação. Como já mencionado, a boa condição nutricional pós-parto do rebanho resultou em alta produção de leite na primeira lactação, mesmo quando as novilhas pariram cedo.

IEP e PP mostraram $r_{g}$ positiva, sugerindo que a seleção para aumento do peso ao primeiro parto resulta em seleção para intervalo de partos mais prolongado, isto é, antagonismo entre peso ao parto e fertilidade após o primeiro parto. A $r_{g}$ entre IEP e PL foi baixa e negativa, -0,26. Ela é esperada devido ao antagonismo entre a PL e características reprodutivas que geralmente ocorrem em rebanhos de alto potencial genético de produção.

O conhecimento da herdabilidade das características envolvidas no processo de produção de leite e a associação genética entre elas são importantes e se justificam pela possibilidade de serem utilizadas na seleção. Elas auxiliam o produtor na tomada de decisões relativas ao manejo da propriedade. Uma forma prática de tirar o máximo proveito dessas informações seria o uso de um índice de seleção em que se estabeleceria um peso específico para cada característica, possibilitando obter progresso na produção de leite sem afetar outras características que façam parte do índice. Como exemplo citam-se o intervalo de partos e o peso ao parto. 


\section{CONCLUSÕES}

O desenvolvimento de novilhas medido pelo peso e pela altura pode contribuir para a obtenção de bons coeficientes técnicos. As herdabilidades obtidas para peso ao primeiro parto, produção de leite na primeira lactação e idade ao primeiro parto indicam que essas características podem ser melhoradas por meio de seleção, enquanto que a estimativa obtida para o primeiro intervalo de partos confirma que essa característica é predominantemente influenciada pelos efeitos ambientais. A seleção para aumento de peso ao primeiro parto pode resultar em aumento no primeiro intervalo de partos, enquanto que a redução da idade ao primeiro parto, provavelmente, não influencia a produção de leite na primeira lactação e sugere um provável antagonismo com o primeiro intervalo de partos.

\section{AGRADECIMENTOS}

Os autores agradecem a FAPESP e ao CNPq pelo apoio financeiro a execução desta pesquisa, e em especial aos responsáveis pelo sistema de produção analisado.

\section{REFERÊNCIAS BIBLIOGRÁFICAS}

ALBUQUERQUE, L.G.; KEOWN, J.F.; VAN VLECK, L.D. Genetic parameters of milk, fat and protein yields in the first tree lactations using an animal model and restricted maximum likelihood. Braz. J. Genet., v.19, p.79-86, 1996.

BOLDMAN, K.G.; KRIESE,L.A.; VAN VLECK, L.D. et. al. Manual for use for MTDFREML: a set of programs to obtain estimates for variances and covariances (DRAFT). Lincoln: Department of Agriculture. Agriculture Research Service, 1995.

CARDOSO, V.L. Efeitos de fatores genéticos e de ambientes sobre o desempenho reprodutivo de vacas da raça Holandesa. 1985. 64f. Dissertação (Mestrado) - Faculdade de Medicina, Universidade de São Paulo, Ribeirão Preto, SP.

DESHMUKH, D.P.; CHAUDHARI, K.B.; DESHPANDE, K.S. Nongenetic and genetic factors affecting production efficiency traits in Jersey, Sahiwal and Jersey $\times$ Sahiwal crossbred cows. Indian Dairy Sci., v.66, p.1128-1147, 1993.

DONG, M.C.; VAN VLECK, L.D. Correlation among first and second lactation milk yield and calving interval. J. Dairy Sci., v.72, p.1933-1936, 1989.

FREITAS, A.F.; DURÃES, M.G.; TEIXEIRA, N.M. Parâmetros genéticos da produção de leite de animais da raça Holandesa mantidos em sistema intensivo de produção tipo free stall. Rev. Bras. Zootec., v.29, supl.1., p.2008-2012, 2000 ,

GADINI, C.H.; KEOWN, J.F.; VAN VLECK, L.D. Parâmetros genéticos da produção de leite, gordura e proteína e escore de células somáticas aos 305 dias de lactação. In: REUNIÃO ANUAL DA SOCIEDADE BRASILEIRA DE ZOOTECNIA, Juiz de Fora, MG. Anais... SBZ, 1997. p.47-49.

GROEN, A.F.; VOS, H. Body weigth and growth in dutch black and white replacement heifers. In: ANNUAL MEETING OF THE EUROPEAN FOR ANIMAL PRODUCTION, 1994, Edinburgh. Proceedings... [s.n.], 1994. p.143.

GROSSI, S.F. Eficiência reprodutiva e produtiva em rebanhos leiteiros monitorados por sistema de informação. 1999. 87f. Dissertação (Mestrado em Zootecnia) - Faculdade de Ciências Agrárias e Veterinárias, Universidade Estadual Paulista, Jaboticabal, SP.

KOENEN, E.P.C.; GROEN, A.F. Genetics analysis to growth patterns to black and white dairy heifers. J. Dairy Sci., v.79, p.495-501, 1995.

MACHADO, S.G.; FREITAS, M.A.R.; GADINI, C.H. Genetic parameters of test day milk yields of Holstein cow. Gen. Molec. Biol., v.22, p.383-386, 1999.

OLIVEIRA, H.N. Análises genético-quantitativa da curva de crescimento de fêmeas da raça Guzerá. 1995. 73f. Tese (Doutorado) - Faculdade de Medicina, Universidade de São Paulo, Ribeirão Preto, SP.

OLIVEIRA, J.A.; BEZERRA, L.A.F.; BASTOS, F.P. Correlações genéticas e fenotípicas entre pesos e produção de leite na primeira lactação em fêmeas da raça Guzerá. Bol. Industr. Anim., v.51, p.07-11, 1994. 
PEIXOTO, A.M. Estudos sobre alguns aspectos de crescimento, eficiência reprodutiva e produção de leite dos mestiços de raça Guernsey em Piracicaba. 1965. Tese (Doutorado). Universidade de São Paulo, Piracicaba-SP.

PEREIRA, J.C.C. Melhoramento genético aplicado à produção animal. 3.ed. Belo Horizonte: FEPMVZ, 2001. 171p.

PEROTTO, D.; CASTANHO, M.J.; ROCHA,J.L. Descrição das curvas de crescimento de fêmeas bovinas Guzerá, Gir, Holandês $\times$ Guzerá e Holandês $\times$ Gir. Rev. Bras. Zootec., v.26, p.283-288, 1997.

PEROTTO, D.; CUE. R.I.; LEE, A.J. Comparison of nonlinear functions for describing the growth curve of three genotypes of dairy cattle. Can. J. Anim. Sci., v.72, p.773-782, 1992.

SILVA, M.V.G.B.; BERGMAN, J.A.G.; MARTINEZ, M.L. Associação genética, fenotípica e de ambiente entre medidas de eficiência reprodutiva da produção de leite na raça Holandesa. Rev. Bras. Zootec., v.27, p.11151122, 1998.

USER'S guide: statistics. Cary, NC: SAS Institute, 1995.

van AMBURGH, M.E.; GALTON, D.M.; BAUMAN D.E. et al. Effects of three prepubertal body growth rates on performance of Holstein heifers during first lactation. J. Dairy Sci., v.81, p.527-538, 1998.

von BERTALANFFY, L. Quantitative laws in metabolism and growth. Q. Review Biol., v.32, p.217-230, 1957.

ZAMBIANCHI, A.R.; FREITAS, M.A.R.; PEREIRA, C.S. Aspectos genéticos e de ambiente da produção de leite e do intervalo entre partos em rebanhos leiteiros monitorados por sistema informação. Rev. Bras. Zootec., v.28, p.1280-1284, 1999. 